\title{
Diferencias en el desarrollo cognitivo y socioemocional según sexo*
}

\author{
Cognitive and Social-emotional Development Differences according to Sex
}

\section{Diferenças no desenvolvimento cognitivo e socioemocional segundo o gênero}

\author{
María Elena Mathiesen, ${ }^{\mathrm{a}}$ Ginette Castro Yánez, ${ }^{\mathrm{b}}$ José Manuel Merino, ${ }^{\mathrm{c}}$ Olga Mora \\ Mardones, ${ }^{\mathrm{d}}$ Gracia Navarro Saldaña ${ }^{e}$
}

${ }^{a}$ Universidad de Concepción, Facultad de Ciencias Sociales, Departamento de Sociología y Antropología. Casilla 160-C. Correo 3. Concepción, Chile. Correo electrónico: mmathies@udec.cl

${ }^{b}$ Universidad de Concepción, Facultad de Humanidades y Arte, Departamento de Idiomas Extranjeros. Telf.: (56-41) 2661380. Correo electrónico: gcastro@udec.cl

'Universidad de Concepción, Facultad de Ciencias Sociales, Departamento de Sociología y Antropología. Concepción, Chile. Telf: (56-41) 2203044. Correo electrónico: jmerino@udec.cl

${ }^{\mathrm{d} U n i v e r s i d a d ~ d e ~ C o n c e p c i o ́ n, ~ F a c u l t a d ~ d e ~ C i e n c i a s ~ S o c i a l e s, ~ D e p a r t a m e n t o ~ d e ~ T r a b a j o ~ S o c i a l . ~ T e l f .: ~(56-41) ~}$ 2204403. Correo electrónico: omora@udec.cl

eUniversidad de Concepción, Facultad de Ciencias Sociales, Departamento de Psicología. Telf.: (56-41) 2203037. Correo electrónico: gnavarro@udec.cl

\begin{abstract}
El propósito del trabajo fue comparar por sexo el desarrollo cognitivo y socioemocional de 100 estudiantes chilenos que pertenecían al programa de Talentos UdeC y que cursaban en su mayoría el último año de enseñanza media. En el año 2004, los alumnos habían sido preseleccionados por sus profesores para participar en tal programa sobre la base de su talento académico. Durante el 2010, se les midió y comparó rendimiento escolar, comprensión lectora, logro en matemáticas, puntajes en la Prueba de Selección Universitaria (PSU), motivación académica, adaptación socioemocional, responsabilidad social, comportamiento social y conducta prosocial y antisocial. Se encontrón diferencias en el desarrollo socioemocional que favorecen a las mujeres. En el ámbito cognitivo, solo se observaron diferencias significativas en dos de los dieciséis indicadores; finalmente, se pudo concluir que no se presentan diferencias según sexo en los ámbitos estudiados.
\end{abstract}

RESUMEN

Palabras clave: diferencias por sexo, talento, desarrollo cognitivo, desarrollo socioemocional.

\begin{abstract}
This study presents a comparison by sex of the cognitive and social-emotional development of 100 Chilean students who participated in the Program Talentos UdeC and the majority of whom are finishing their senior year of high school. These students were pre-selected in 2004 by their teachers to participate in the Program based on their academic talent. In 2010, their academic performance, reading comprehension, mathematical level, university selection standardized test scores (PSU), academic motivation, social-emotional adaptation, social responsibility, and pro-social /antisocial behavior were measured and compared. Our results showed differences in social-emotional development, where women were favored. Significant differences in cognitive development were observed in only two of the 16 indicators. Based on our results, we concluded that there were no significant differences between sexes en the areas studied.
\end{abstract}

Key words: sex differences, talent, cognitive development, socio-emotional development.

* $\quad$ Este artículo se inserta en el Proyecto Fondecyt 1100260 - "Incubando capital humano regional: evaluación longitudinal de un programa extracurricular universitario para estudiantes de establecimientos municipales de alto potencial académico" (2010-2011). Consultas y sugerencias a: mmathies@udec.cl o correo postal Víctor Lamas 1290, casilla 160-C, Correo 3, Concepción, Chile. 


\section{RESUMO}

Comparou-se, segundo o gênero, o desenvolvimento cognitivo e socioemocional de 100 estudantes chilenos, em maioria, cursando o último ano do Ensino Médio e pertencentes ao programa de Talentos UdeC. No ano de 2004, Tais alunos, por causa do bom desempenho acadêmico, haviam sido selecionados por seus professores para participar do programa. Durante o ano de 2010, foram avaliados e comparados o rendimento escolar, a compreensão da leitura, o êxito em matemática, os pontos na Prova de Seleção Universitária (PSU), a motivação acadêmica, a adaptação socioemocional, a responsabilidade social, o comportamento social e a conduta social e antissocial. Foram encontradas diferenças no desenvolvimento socioemocional que favoreceram o gênero feminino. No âmbito cognitivo, somente foram observadas diferenças significativas em dois dos dezesseis indicadores; finalmente, concluiu-se que, segundo o gênero, não se apresentam diferenças nos âmbitos estudados.

Palavras chave: diferenças por gênero, talento, desenvolvimento cognitivo, desenvolvimento socioemocional.

\section{INTRODUCCIÓN}

Una de las áreas más estimulantes en la ciencia social actual tiene que ver con las diferencias entre hombres y mujeres. Dos grandes enfoques se enfrentan en esta área: el enfoque más popular y prevalente entre los científicos sociales es aquel que atribuye estas diferencias a la socialización de género. De acuerdo a esta explicación, hombres y mujeres (y niños y niñas) piensan y se comportan diferente porque han sido socializados en forma distinta por sus culturas y sociedades. Un enfoque sociobiológico muy contemporáneo, de la denominada psicología evolucionista, postula algo distinto: hombres y mujeres son diferentes no porque hayan sido socializados de manera diversa, sino porque sus diferencias en conducta, cogniciones, valores y preferencias son parte de la naturaleza humana femenina y masculina distintas desde el nacimiento. La socialización de género ayuda a acentuar, solidificar, perpetuar y fortalecer esas diferencias innatas entre hombres y mujeres, pero no a causar o crearlas. En otras palabras, hombres y mujeres no son diferentes porque sean socializados diferentemente, sino que más bien son socializados de manera distinta porque son distintos. La socialización de género no es la causa de la diferencia de sexo, es más bien su consecuencia (Barkow, Cosmide, y Tooby, 1992; Freese y Powell, 1999; Kanazawa y Vandermassen, 2005; Gintis, 2007; Miller y Kanazawa, 2007).

Cuando se comparan hombres y mujeres es muy difícil separar diferencias que se deben a la influencia del medio cultural en que se desarrollaron respecto de aquellas específicamente biológicas. Así, además de las evidentes diferencias debidas al dimorfismo sexual hay otras, como por ejemplo, en la formación cerebral: el cuerpo calloso que comunica los hemisferios cerebrales es más ancho en las mujeres que en los hombres, lo que facilita una mayor comunicación en el procesamiento del lenguaje, y el núcleo del hipotálamo es 2.5 veces más grande en los hombres que en las mujeres, lo que influye en la regulación de las emociones e impulsos sexuales (Acarín y Acarín, 2002; Fernández, 2003). Rutter (1970, Cit. en Grimm, 2003: 69) por su parte informa de diferencias de maduración que favorecerían a las niñas desde el nacimiento y que podrían acentuarse llegando a ser, en algunos casos, de hasta dos años durante la pubertad; desde allí la brecha en el desarrollo comenzaría a desaparecer.

El interés por analizar las diferencias de sexo en el desarrollo cognitivo y psicosocial en estudiantes, surge de nuestra experiencia en investigación sobre calidad educativa y desarrollo infantil. Allí hemos observado que las niñas ingresan a jardines infantiles con promedios de desarrollo léxico (Herrera, Mathiesen y Pandolfi, 2000) o motriz y 
léxico (Morales, Mathiesen y Navarro, 2008) significativamente más altos que los niños y terminan el año con promedios inferiores a éstos, asunto que podría explicarse por una maduración más precoz de las niñas (Bethencourt y Torres, 1987) o por el ambiente educativo, como lo informan otros autores. Bing (1963) muestra cómo las prácticas de crianza podrían explicar la mayor habilidad espacial y matemática de los varones y su menor habilidad en lenguaje. En este mismo sentido, apoyados en diversos autores, Bethencourt y Torres (1987) afirman que las diferencias en los resultados de los niños y las niñas se explicarían porque las expectativas de padres y maestros serían más altas para los niños. Steinmayr y Spinath (2009), por su parte, encontraron que las expectativas de los padres explicarían la mayor inteligencia y confianza en ésta de los niños en relación con las niñas.

En América Latina y El Caribe la existencia de diferencias de desarrollo entre un niño y una niña depende del tipo de población, la condición educativa, el nivel socioeconómico y la calidad de las instituciones educativas a la que acceden (Organización de las Naciones Unidas para la Educación, la Ciencia y la Cultura [UNESCO], 2003). De esta manera, se estableció que una persona tiene menos oportunidades de desarrollo si es mujer, si vive en contextos de pobreza, si pertenece a una etnia indígena y si tiene una lengua materna indígena, por lo tanto, un grupo que tenga una o más de estas características tendrá que ser prioridad para favorecer sus potencialidades educativas de acuerdo a sus posibilidades, intereses y habilidades (Programa de Promoción de la Reforma Educativa en América Latina y El Caribe [PREAL], 2004).

En Chile se han realizado estudios que muestran diferencias significativas entre el desarrollo de niños y niñas. Un estudio desarrollado en centros educativos de la Fundación Nacional para el Desarrollo Integral del Menor (Integra, 2005) que atiende a los niños y niñas más pobres de Chile de cinco y menos años de edad, concluyó que existen diferencias cognitivas significativas entre ellos, mostrando los niños mayores puntajes que las niñas (Integra, 2005). En otra investigación Herrera et al. (2000) informan de resultados pobres en lenguaje en niños y niñas de sectores socioeconómicos bajos y son los varones los que obtienen puntajes significativamente más altos. Otro estudio que observó salas de actividades, determinó que existía discriminación de género del adulto a cargo hacia los niños y niñas de tres y menos años de edad, que favorecía a los niños y perjudicaba a las niñas (Herrera, Mathiesen, Morales, Proust y Vergara, 2006). En definitiva las desigualdades de género respecto a las oportunidades de aprendizaje y desarrollo van en desmedro de las niñas (PREAL, 2004).

Las diferencias de rendimiento están estrechamente ligadas con las desigualdades sociales, las que se pueden dar a través de la organización institucional, del tipo de interacción, de los métodos pedagógicos, de las creencias docentes, y a través de los materiales y recursos económicos de la institución (De Oliveira, 2004). Algunos estudios señalan que los adultos a cargo de menores interactúan de manera diferente con los niños y con las niñas, les hablan más a los niños que a las niñas, refuerzan positivamente más a los varones y son más permisivos con éstos y usan un mayor número de justificaciones estereotipadas con las niñas (Herrera et al., 2006). Ahora bien, aludiendo al rol de los profesores, éstos formulan una mayor cantidad de preguntas divergentes a los niños, mientras que a las niñas se las interroga sobre aspectos más bien académicos. También, los docentes al realizar críticas hacia el trabajo de niños y niñas, acuden a atributos 
personales en el caso de las niñas y, en el caso de los niños, a la calidad del trabajo (Lera y Sánchez, 2001).

Se han establecido metas a nivel internacional, las que señalan que para el año 2015, Chile debe lograr la igualdad de género en cobertura y oportunidades educativas en todos los niveles de la educación (Organización de las Naciones Unidas para la Educación, la Ciencia y la Cultura [UNESCO], 2004).

En el contexto de nuestro trabajo en el Programa de Talentos UdeC hemos visto que los profesores preseleccionan entre un 5 y un $10 \%$ más de niños que de niñas, lo que nos ha inquietado acerca del tema de las diferencias de género. Así en este artículo nos planteamos la pregunta: en estudiantes seleccionados por sus profesores como con posible talento académico cuando cursaban sexto básico (2004), ¿existen diferencias de desarrollo cognitivo y psicosocial según sexo al finalizar su enseñanza media (2010)?

\section{MÉTODO}

\subsection{MUESTRA}

La muestra teórica fue de 120 alumnos, se planificó en el marco de una investigación cuasi experimental que pretende evaluar el efecto de un programa educativo para niñas, niños y jóvenes con talento académico destacado de la Universidad de Concepción (Talentos UdeC). Todos los alumnos y alumnas fueron preseleccionados para participar en el Programa Talentos UdeC en el año 2004, cuando cursaban sexto año en colegios municipales. La muestra fue conformada por 40 estudiantes que habían ingresado y asistido a lo menos tres años al programa, los que constituyen el grupo tratamiento. Se seleccionaron además dos grupos controles, uno de 40 alumnos de inteligencia similar al grupo tratamiento (con puntaje de corte o hasta 2 puntos por debajo de éste) y otro grupo control de inteligencia normal (entre el percentil 40 y el 60). La inteligencia fue medida con el test de Matrices Progresivas Raven. En los dos primeros grupos se incluyó a todos los sujetos que cumplían con los requisitos muestrales. En el caso del grupo control 2, se seleccionó a azar a la mitad de los sujetos que tenían las características requeridas (se tomaron 6 más previendo pérdidas). Se seleccionó 126 alumnos de un listado del 2004, de la base de datos de Talentos UdeC. La muestra real quedó constituida por 100 alumnos: 37 del grupo tratamiento, 36 del grupo control 1 y 27 del grupo control 2. Las pérdidas se debieron a rechazos en la participación de directores de establecimientos educacionales, a alumnos que no aceptaron rendir las pruebas y a alumnos imposibles de ubicar por cambios de colegio o residencia.

Respecto al sexo que es la variable central de este trabajo, como se puede apreciar en la Tabla 1, hay una proporción mayor de hombres. Las mayores diferencias se dan en el grupo control 2 en el que predominan las mujeres, sin embargo, estas diferencias no son significativas $(\mathrm{Chi}=3.17, \mathrm{p}<0.21)$. 
Tabla 1. Integrantes de la muestra por sexo y grupo

\begin{tabular}{|c|c|c|c|}
\hline Grupo & Mujeres & Hombres & Totales \\
\hline Tratamiento & 16 & 21 & 37 \\
\hline Control 1 & 17 & 19 & 36 \\
\hline Control2 & 7 & 20 & 27 \\
\hline Totales & 40 & 60 & 100 \\
\hline
\end{tabular}

La edad presenta una fluctuación entre 15 y 19 años, con un promedio de 17,44 años. Casi todos los participantes cursaban $4^{\circ}$ medio $^{1}(94 \%)$, el $5 \%$ cursaba $3^{\circ}$ medio y sólo un alumno cursaba $1^{\circ}$ y $2^{\circ}$ medio simultáneamente. El promedio de edad de las mujeres fue de 17,52 años con una desviación de 0.5, un mínimo de 17 y un máximo de 18; el promedio de edad de los hombres fue de 17,23 con una desviación de 0.6 y fluctúo entre 15 y 19, sin diferencias significativas $(\mathrm{t}=1.21, \mathrm{p}<.23)$. Tampoco hubo diferencias significativas en las proporciones por sexo, si se tiene en cuenta el tipo de sostenedor del colegio, a pesar del $15 \%$ más de hombres que asistían a establecimientos particulares subvencionados $(\mathrm{Chi}=2.92, \mathrm{p}<0.23)$. La proporción de mujeres en enseñanza científico humanista fue un $19 \%$ mayor que la de hombres, diferencia que tampoco alcanzó significación $(\mathrm{Chi}=4.50,<0.11)$.

En relación al establecimiento educacional, durante el año 2004 todos eran alumnos de colegios municipales de las cuatro comunas del gran Concepción (Concepción, Talcahuano, San Pedro y Chiguayante). Durante el 2010 sólo 49\% asistía a este tipo de establecimientos, $29 \%$ iba a colegios particulares subvencionados, el $20 \%$ a colegios de Fundaciones Educacionales y el $2 \%$ a un colegio particular pagado (alumnos que fueron reubicados después del terremoto de febrero de 2010).

Las comunas en que funcionaban estos establecimientos eran las mismas del 2004, pero se agregaron Hualpén (comuna nueva) y Coronel; sin embargo, la mayoría de los integrantes de la muestra asistían a colegios de Concepción (54\%), Talcahuano (22\%) o Hualpén (20\%); en las otras comunas había pocos alumnos, con cifras que fluctúan entre 3 y 9\%. En cuanto a la comuna de residencia, la mayoría vivía en Talcahuano (28\%), le siguen Concepción y Hualpén con el $20 \%$ cada una y San Pedro con el 17\%. Por tanto, estos alumnos prefieren Concepción para estudiar, a pesar de vivir en otras comunas. En cuanto al tipo de establecimiento, mayoritariamente asistían a liceos científico-humanistas $(61 \%)$, le siguen los liceos comerciales $(20 \%)$, los técnicos $(11 \%)$ y una pequeña cantidad a liceos industriales $(6 \%)$ o polivalentes $(2 \%)$.

\subsection{INSTRUMENTOS}

A continuación se describen brevemente los instrumentos que fueron aplicados a toda la muestra, que se dividen en dos grandes grupos: Desarrollo Cognitivo y Desarrollo Socioemocional.

1 En Chile la escolaridad obligatoria es de 12 años, los ocho primeros constituyen la educación básica y los cuatro últimos, la enseñanza media. 


\section{Desarrollo Cognitivo}

Test de Matrices Progresivas (Raven, 2003): Se trata de la adaptación argentina de 1993 de este test de inteligencia. Se presentan 60 láminas de dibujos con una porción faltante, distribuidos en cinco series con doce dibujos cada una, el sujeto debe elegir la que corresponde de entre seis y ocho alternativas de respuesta. Cada serie comienza con un problema fácil de resolver y se va dificultando la elección hasta llegar al número doce, cada respuesta correcta corresponde a un punto, por tanto, el máximo posible es de 60 puntos. Existen tablas por edades para ubicar el percentil que le corresponde a cada sujeto evaluado. En este caso, hemos usado los puntajes disponibles de un estudio mayor, obtenidos en el año 2004 durante el proceso de selección al programa Talentos UdeC.

Prueba de Matemáticas: Para la construcción de esta prueba se seleccionaron 14 ejercicios de diversos ensayos PSU (Prueba de Selección Universitaria) de matemáticas. Se realizó una prueba piloto, con el objeto de validar el test resultante que se aplicó en un colegio particular de la región del Maule a 16 alumnos de $4^{\circ}$ medio. Hubo dos ítems que presentaron problemas que fueron suprimidos quedando la prueba con 12 ejercicios. Luego de aplicada se realizó un análisis psicométrico todos los ítems correlacionaron significativamente con la escala total. La confiabilidad fue adecuada, se obtuvo un alfa de Cronbach de 0.74. En cuanto a la validez, ésta también resultó adecuada, pues hubo una correlación de 0.76 con una $\mathrm{p}<0.0001$ con la PSU en matemáticas.

Test de Comprensión Lectora. Para la elaboración de esta prueba se recopiló una serie de 40 ítems con los respectivos textos de los cuales emergieron. Estos ítems habían sido utilizados en pruebas PSU anteriores o en ensayos de distintos institutos por lo que habían sido probados en su eficiencia. Medían tanto comprensión global, como específica e inferencial. Con el conjunto de textos y sus preguntas se elaboró dos pruebas piloto alternativas que fueron aplicadas, en dos vueltas, a 10 sujetos de primer año de universidad de una carrera de humanidades. Una vez analizados los resultados, se procedió a eliminar los ítems de mucha dificultad y muy largos. Finalmente, la prueba que se aplicó quedó constituida por 4 textos y 12 preguntas.

Test de Vocabulario en Imágenes, TEVI-R (Echeverría, Herrera y Segure, 2009). Este test de vocabulario en imágenes mide el nivel de comprensión de vocabulario pasivo que posee un sujeto. Puede ser utilizado con niños de edades que fluctúan entre dos años y medio y 19 años. En su aplicación se requiere que el sujeto señale una de las cuatro láminas que se le presentan mediante estímulo auditivo ofrecido por el entrevistador. El test se encuentra estandarizado para Chile desde 1982 y permite comparar el resultado con una norma por edad. Se utilizó la versión revisada, disponible desde el año 2002.

Notas de la Enseñanza Media (NEM). De los registros de los establecimientos educacionales se obtuvo los promedios de notas de las asignaturas de Lenguaje y Matemáticas así como también los promedios generales desde el año 2007 al año 2009.

Puntajes Prueba de Selección Universitaria (PSU). La PSU es un conjunto de pruebas que viene siendo aplicado por el Consejo de Rectores de Universidades Chilenas desde el año 2003. Estas pruebas han sido sometidas a estudios que demuestran su validez predictiva, siendo la prueba de Matemáticas junto con el puntaje que pondera NEM (Notas Enseñanza Media) los mejores predictores del rendimiento académico en la Universidad (Consejo de Rectores Universidades Chilenas, 2006). Sus resultados se publican en Internet, de estos listados oficiales se obtuvieron los puntajes de los integrantes de la 
muestra que se informan en este trabajo que son NEM y PSU Matemáticas y Lenguaje. No se incluyen los puntajes PSU de Ciencias Sociales ni de Ciencias Naturales debido al escaso número de estudiantes que las rindieron.

\section{Desarrollo Socioemocional.}

Cuestionario de Adaptación Socioemocional: Se trata de una escala tipo Likert, construida por el equipo Talentos UdeC a partir de los resultados de una investigación cualitativa realizada en una muestra de 10 psicólogos clínicos infantojuveniles, que se desempeñaban profesionalmente en la provincia de Concepción y a quienes se les consultó por conductas que reflejan adaptación socioemocional y que sean posibles de ser autoreportadas. Se seleccionó comportamientos de las tres áreas a las que los escolares deben adaptarse: la escolar, la familiar y la personal, y que además reflejaran habilidades sociales, emocionales y cognitivas. La escala original que quedó formada por 27 ítems, con 5 alternativas de respuesta (totalmente en desacuerdo, un punto; en desacuerdo, dos; ni de acuerdo ni en desacuerdo, tres; de acuerdo, cuatro y totalmente de acuerdo, cinco). Posterior a un estudio psicométrico (Mathiesen, Castro, Merino, Mora y Navarro, 2011) se suprimieron 4 ítems, por tanto, la escala quedó constituida por 23 ítems distribuidos en tres subescalas: Habilidades emocionales, con 10 ítems, Percepción de Autoeficacia, con 7 ítems y Habilidades Sociales, con 5 ítems. Del total de 13 ítems invertidos del formato original quedaron 10 en la escala final. Se da cuenta de un alfa de Cronbach para la escala total de 0.84 y de 0.71 para autoeficacia, 0.74 y 089 para habilidades sociales y emocionales, respectivamente.

Cuestionario de Motivaciones e Intereses: Este instrumento también fue elaborado por el equipo de sicólogos del Programa Talentos UdeC. Se trata de una escala Likert de 28 ítems, sobre los que el encuestado debe manifestar su grado de acuerdo, desde el total acuerdo (5 puntos) hasta el total desacuerdo (1 punto). Se hizo un estudio psicométrico que demuestra la confiabilidad de este cuestionario con un alfa de Cronbach de 0.88 y correlaciones altamente significativas de todos sus ítems con el total de la escala.

Cuestionario de Comportamientos Socialmente Responsables: Se trata de un listado de 19 comportamientos que muestran responsabilidad social sobre los que el sujeto debe informar la frecuencia con que los realiza desde nunca ( 0 puntos) hasta siempre (4 puntos). También se hizo un estudio psicométrico de este cuestionario que demuestra su confiabilidad con un alfa de Cronbach de 0.82 y correlaciones altamente significativas de todos sus ítems con el total de la escala.

Cuestionario de Comportamiento Social: (Obtenido de Texas Social Behavior Inventory de Helmreiech, Stapp y Ervin (1974), en Robinson y Shaver, 1991). Se trata de un cuestionario de 16 ítems con dos formatos. En este caso se aplicó el formato A que tiene seis ítems invertidos. Los participantes deben manifestar en qué medida los representan una serie de afirmaciones desde "No me representa para nada" (1 punto) hasta "Me representa completamente" (5 puntos) pasando por "No mucho" "Algo" y "Bastante" con 2, 3 y 4 puntos, respectivamente. Es un instrumento corto, de fácil comprensión y respuesta. El estudio psicométrico realizado mostró una confiabilidad adecuada con un alfa de $0.77 \mathrm{y}$ sólo un ítem que no correlacionó significativamente con el total. Los autores informan adecuados resultados psicométricos para sus aplicaciones en EE.UU.; el estudio psico- 
métrico realizado por nosotros, mostró una confiabilidad adecuada con un alfa de 0.77 y sólo un ítem que no correlacionó significativamente con el total.

Inventario de Conductas Sociales: (Obtenido de Teenage Inventory of Social Skills (TISS) de Inderbitzen y Foster, 1992, citado y reproducido en Inderbitzen y Harbin, 1992). La versión en español de este inventario se obtuvo de Roa (2010). Se trata de un cuestionario de tipo Likert, de 40 afirmaciones, 20 de comportamientos prosociales y 20 antisociales, sobre las que el encuestado debe manifestar en qué grado tiene estos comportamientos en el colegio. Puntúa desde "nada" (1 punto) hasta "muchas veces" (5 puntos), pasando por "poco", "a veces" y "varias veces" con 2, 3 y 4 puntos, respectivamente. Revirtiendo los ítems, la escala puede usarse con los 40 ítems como de comportamiento prosocial total y/o como una escala prosocial y otra antisocial, cada una de 20 ítems. Con el estudio sicométrico realizado se encontró alfas de 0.78 para la escala total y para la de comportamiento antisocial, mientras que para la de comportamiento prosocial fue de 0.85 . La escala total tuvo 4 ítems que no correlacionaron con el total, mientras que ambas subescalas con todos sus ítems tuvieron todas las correlaciones significativas. Es por esto que recomendamos usar ambas subescalas en lugar de la escala total.

\section{PROCEDIMIENTO}

Entre julio y agosto del año 2010 se obtuvo las autorizaciones de la mayoría de los Directores de establecimientos educacionales y los resultados académicos de los integrantes de la muestra, sacados de los registros institucionales. Luego, a partir de agosto, se siguió trabajando con los participantes que faltaban. Además, se obtuvo los asentimientos de los alumnos y, a través de ellos, los consentimientos de los padres. Los instrumentos se aplicaron entre agosto y noviembre en salas de los establecimientos educacionales y, en el caso de algunos alumnos cuyos colegios no dieron la autorización, ellos aceptaron participar y la aplicación se realizó en salas de la Universidad. Se aplicaron 3 pruebas de Desarrollo Cognitivo y 5 de Desarrollo Socioemocional. Se obtuvieron los promedios de notas de desde el año 2007 al 2009 y en diciembre, los puntajes PSU y NEM. Se digitaron datos y se construyó una base SAS utilizando el programa correspondiente (Statistical Analysis System, versión 9.1.2; Licencia Universidad de Concepción - Escuela de Graduados, SITE 0050543001).

\section{RESULTADOS}

\subsection{ASPECTOS COGNITIVOS Y GÉNERO}

Como se puede apreciar en la Tabla 2, la inteligencia, evaluada el 2004, presenta un promedio ligeramente mayor en las mujeres que en los hombres, diferencia que no alcanza significación estadística. En relación con el rendimiento académico global, las mujeres presentan promedios ligeramente más altos que los hombres, diferencias que solo alcanzan significación en el rendimiento del año 2008. 
Tabla 2. Comparación de promedios de Desarrollo cognitivo según género

\begin{tabular}{|c|c|c|c|c|}
\hline Variable & $\overline{\mathrm{x}}$ mujeres & $\overline{\mathrm{x}}$ hombres & t de Student & $\mathbf{p}$ \\
\hline Inteligencia 2004 & 48.58 & 47.92 & 1.18 & 0.24 \\
\hline$\overline{\mathrm{x}}_{2007}$ & 5.98 & 5.54 & 1.19 & 0.24 \\
\hline$\overline{\mathrm{X}} 2008$ & 6.07 & 5.82 & 2.15 & 0.03 \\
\hline$\overline{\mathrm{X}} 2009$ & 6.05 & 5.93 & 1.13 & 0.28 \\
\hline NEM & 628.59 & 610.21 & 0.82 & 0.42 \\
\hline Matemáticas & 46.04 & 569.49 & 0.50 & 0.62 \\
\hline PSU Matemáticas & 569.49 & 574.20 & 0.27 & 0.79 \\
\hline$\overline{\mathrm{x}}$ Matemáticas 2007 & 5.63 & 5.62 & 0.10 & 0.90 \\
\hline$\overline{\mathrm{X}}$ Matemáticas 2008 & 5.75 & 5.55 & 0.66 & 0.51 \\
\hline$\overline{\mathrm{x}}$ Matemáticas 2009 & 5.85 & 5.55 & 2.09 & 0.04 \\
\hline Comprensión Lectora & 46,3 & 40,5 & 1.71 & 0.09 \\
\hline PSU lenguaje & 559.65 & 565.57 & 0.33 & 0.74 \\
\hline$\overline{\mathrm{X}}$ Lenguaje 2007 & 5.69 & 5.75 & 0.35 & 0.73 \\
\hline$\overline{\mathrm{X}}$ Lenguaje 2008 & 5.70 & 5.58 & 0.62 & 0.54 \\
\hline$\overline{\mathrm{X}}$ Lenguaje 2009 & 5.93 & 5.72 & 1.18 & 0.24 \\
\hline Vocabulario & 94.20 & 97.13 & 0.84 & 0.40 \\
\hline
\end{tabular}

En Matemáticas, los resultados fueron similares, ligeramente más altos en las mujeres que en los hombres salvo en la PSU; la única diferencia significativa en Matemáticas, es la del año 2009, donde se muestra un promedio significativamente mayor en las mujeres. Respecto al Lenguaje, tampoco hay diferencias significativas entre hombres y mujeres aunque aquí hay más diversidad de resultados. Así, por ejemplo, la Comprensión Lectora arrojó resultados algo más altos en las mujeres. El promedio PSU Lenguaje de los hombres fue ligeramente más alto que el de las mujeres. El año 2007 los hombres están levemente mejor en Lenguaje, mientras que en el año 2008 la situación se revierte, ya que las mujeres tienen un promedio algo más alto que los hombres. Finalmente, en el año 2009 las diferencias aumentan a favor de las mujeres en este ámbito. No obstante, en Vocabulario los hombres tienen 3 puntos más. En síntesis, se aprecia que los resultados en todos los indicadores que se utilizaron para evaluar lenguaje son similares en mujeres y hombres.

\subsection{ASPECTOS SOCIOEMOCIONALES Y GÉNERO}

Como se puede observar en la Tabla 3, en motivación académica y responsabilidad social se aprecia la misma tendencia, que no alcanza significación. Existe mayor motivación y responsabilidad en las mujeres que en los hombres. La Adaptación socioemocional total, social y emocional, es significativamente mayor en las mujeres. En la percepción de autoeficacia la significación desaparece, esto es, las mujeres tienen más habilidades 
sociales y emocionales que los hombres y no habría diferencias de género respecto de la autoeficacia, al menos entre los participantes de este estudio.

Tabla 3. Comparación de promedios de Desarrollo Socioemocional según género

\begin{tabular}{|c|c|c|c|c|}
\hline Variable & $\overline{\mathrm{X}}$ mujeres & $\overline{\mathrm{X}}$ hombres & T de Student & $\mathbf{P}$ \\
\hline Motivación académica & 4.33 & 4.23 & 1.21 & 0.23 \\
\hline Responsabilidad social & 2.55 & 2.42 & 1.61 & 0.11 \\
\hline $\begin{array}{c}\text { Adaptación } \\
\text { socioemocional }\end{array}$ & 4.01 & 3.78 & 2.20 & $0.03^{*}$ \\
\hline Autoeficacia & 4.14 & 4.11 & 0.28 & 0.78 \\
\hline Adaptación social & 3.45 & 3.12 & 2.02 & $0.05^{*}$ \\
\hline Adaptación emocional & 3.45 & 3.12 & 2.72 & $0.008^{* *}$ \\
\hline Comportamiento social & 3.57 & 3.50 & 0.70 & 0.49 \\
\hline Conducta prosocial & 4.08 & 3.74 & 3.40 & $0.001^{* *}$ \\
\hline Conducta antisocial & 2.10 & 2.37 & 3.25 & $0.002^{* *}$ \\
\hline
\end{tabular}

Los resultados de Comportamiento social no muestran diferencias significativas aunque el promedio es mayor en las mujeres. En Conducta prosocial las mujeres obtienen un promedio significativamente mayor que los hombres mientras que en Conducta antisocial son los hombres quienes presentan el promedio significativamente mayor. Por tanto, se puede afirmar que las mujeres de esta muestra tienen mayor conducta prosocial y los hombres más conducta antisocial (ver tabla 3).

\section{DISCUSIÓN Y CONCLUSIONES}

Los resultados muestran que en aspectos cognitivos, prácticamente no hay diferencias significativas según sexo en la muestra analizada. En el año 2008, las mujeres obtuvieron un promedio global de notas significativamente más alto que los hombres y el 2009 un promedio de rendimiento en Matemáticas también significativamente más alto que los hombres. En el resto de los indicadores de habilidad matemática, las mujeres obtienen promedios ligeramente superiores a los de los hombres, pero sin significación estadística, lo que sería concordante con Hyde y Mertz (2009) quienes, en un estudio en los Estados Unidos, encontraron que las niñas se desempeñan tan bien como los varones en pruebas estandarizadas de Matemáticas. En Lenguaje no hay diferencias entre hombres y mujeres. Esta falta de diferencias según sexo en el Desarrollo cognitivo podría deberse a que en alumnos con talento académico, realmente estas no existen o podrían explicarse, por una parte, por un sesgo en la preselección, pues los profesores el año 2004 eligieron un 10\% más de hombres, lo que significa que los profesores habrían sido más exigentes para incluir mujeres y, por otra parte, a que la proporción de mujeres de inteligencia normal fue de $18 \%$ frente a un $33 \%$ de hombres, aunque esta diferencia no fue significativa. 
En todo caso, lo encontrado por nosotros difiere a lo informado por la literatura en la que se afirma la superioridad de las mujeres en comunicación y procesos verbales (Acarín y Acarín, 2002; Fernández, 2003). También referido a trabajos verbales de estudiantes, Levy ya en 1972 hipotetiza, desde la neuropsicología, que esta superioridad podría deberse a que las mujeres usan ambos hemisferios cerebrales en funciones de lenguaje, mientras que los hombres usarían solo el izquierdo, reservando el derecho para funciones visoespaciales, lo que explicaría su superioridad en matemáticas (Cit. en Elises, Riveras, González y Crespo, 1990: 127). Esta superioridad tampoco pudo ser detectada en las y los jóvenes de nuestra muestra.

Otros autores también informan de un rendimiento en Matemáticas mayor de los hombres, especialmente en los niveles de más edad, que son los que corresponden a nuestra muestra (Adediwura y Omoyemiju, 2010; Marshall 1980 y 1984, en Bethencourt y Torres, 1987). Por otra parte, Elises et al. (1990), aunque detectaron una tendencia a mayor rendimiento en Lenguaje en las niñas, no encontraron diferencias significativas de rendimiento escolar en una muestra de 505 alumnos y alumnas de enseñanza general básica de Valladolid. Estos últimos resultados serían más concordantes con los detectados en este trabajo. En inteligencia se encontró un promedio mayor de 0.66 puntos más en las mujeres que no alcanzó significación mientras que Nyborg (2005) encontró 3.5 puntos más de inteligencia en hombres que en mujeres.

En general, la literatura respecto al desarrollo cognitivo según el sexo presenta resultados que varían mucho según la edad de los sujetos, la época y la localización del estudio, lo que estaría mostrando tanto la multicausalidad de estos resultados como la complejidad del tema en estudio. En cambio, en desarrollo socioemocional se observa una ventaja de las mujeres comparadas con los hombres en todos los indicadores seleccionados. Así, en esta muestra, tienen significativamente más Adaptación social y emocional y Conducta prosocial, las mujeres que los hombres, y también existe una tendencia clara a mayor motivación académica y mayor responsabilidad social, hallazgos similares a los informados por Inglés, Benavides, Redondo, García-Fernández, Ruiz-Esteban, Estévez y Huescar (2009, 94). También en consonancia con nuestros resultados existen reportes de las ciencias médicas que muestran diferencias entre los cerebros de las mujeres y de los hombres: el lóbulo frontal sería proporcionalmente mayor en las mujeres y allí, en la corteza límbica, se regularían las emociones. Sin embargo, existiría una inconsistencia con lo afirmado por Acarín y Acarín (2002), en el sentido que los hombres estarían mejor dotados que las mujeres para controlar sus emociones y sus impulsos sexuales, por tener el núcleo del hipotálamo más grande, área responsable de regular las emociones e impulsos sexuales.

Por otra parte, hay estudios que explicarían el mejor manejo social de las mujeres por su socialización, ya que desde la infancia e incluso a través de los juguetes, se estarían promoviendo en las niñas habilidades emocionales de cooperación y comprensión (Blanco, 2000; Fisher, 2000). Los niveles de regulación que alcance un niño o niña dependerían principalmente del contexto social en que estén insertos y cómo este contexto los estimule. Por lo mismo, las niñas, a través de la constante estimulación emocional y a pesar de tener un núcleo del hipotálamo más pequeño, logran un mayor desarrollo emocional, mientras que los niños, tienen un menor desarrollo por la menor estimulación de sus potenciales habilidades emocionales. En el caso de los hombres, el ambiente suele estimular comportamientos más agresivos, por lo que sus habilidades para desarrollar y 
regular adecuadamente sus emociones se verían menoscabadas. En los varones de esta muestra se detectó mayor Conducta antisocial, lo que es concordante con lo informado por la literatura (Cabrera, 2002; Garaigordobil, 2005).

Basados en lo anteriormente expuesto podemos responder a nuestra pregunta de investigación que busca establecer si existen diferencias por sexo en el desarrollo cognitivo y psicosocial en estudiantes preseleccionados como con talento académico por sus profesores. Podemos establecer la existencia de diferencias demostrables en desarrollo socioemocional que favorecen a las mujeres. En desarrollo cognitivo, en tanto, los resultados son menos categóricos y se puede concluir que no existen diferencias según sexo. Finalmente, sostenemos la necesidad de realizar más estudios comparativos por sexo y por desarrollo en los distintos niveles educacionales, tanto en muestras representativas de estudiantes chilenos de inteligencia media como de jóvenes con talento académico.

\section{REFERENCIAS BIBLIOGRÁFICAS}

Acarín, N. y Acarín, L. (2002). El cerebro del rey. Barcelona: RBA.

Adediwura, A. y Adeniyi, O. (2010). A Study of Gender Differences in the Attitude of Mathematically Gifted and Non-gifted Senior School Students in Nigeria. Gender \& Behaviour. Ile-Ife, vol. 8, n. 2, 3101-3115.

Barkow, J., Cosmides, L., y Tooby, J. (1992). The Adapted Mind: Evolutionary Psychology and the Generation of Culture. New York: Oxford University Press.

Bethencourt, J. T. y Torres, E. (1987). La diferencia de sexo en la resolución de problemas aritméticos: un estudio transversal. Infancia y aprendizaje, n. 38, 9-20.

Blanco, N. (2000). El sexismo en los materiales educativos de la ESO. Sevilla: Instituto Andaluz de la mujer.

Bing, E. (1963). Effects of child-rearing practices on development of differential cognitive abilities. Child development, vol. 34, n. 3, 631-648.

Cabrera, O. (2002). Psychological and behavioral correlates of adolescent gang involvement. Dissertation Abstracts International: Section B: The Sciences and Engineering, 62 (11-B).

Consejo de Rectores Universidades Chilenas (2006). Estudio acerca de la Validez Predictiva de los Factores de Selección a las Universidades del Consejo de Rectores. Santiago: CRUCh, Documentos Técnicos.

De Oliveira, M. L. (2004). Las diferencias de género y color en las escuelas de Brasil: Los maestros y la evaluación de los alumnos. En: Winkler, D.R. y Cueto, S. (Eds.), Etnicidad, Raza, Género y Educación en América Latina (pp. 373-391). Washington, DC: PREAL.

Echeverría, M.; Herrera, M.O. y Segure, J. (2009). TEVI-R Test de Vocabulario. En Imágenes. Concepción: Editorial Universidad de Concepción, tercera edición revisada.

Elises, J. A., Riveras, F., González, C. y Crespo, M. (1990). El rendimiento escolar en función del sexo al inicio de la enseñanza general básica. Revista Interuniversitaria de Formación del Profesorado, vol. 8, 123-132.

Fernández, F. (2003). Sociología de la Educación. Madrid: Pearson

Freese, J. y Powell, B. (1999). Sociobiology, Status, and Parental Investment in Sons and Daughters: Testing the Trivers-Willard hypotesis. American Journal of Sociology, vol. 104, n. 6, 1704-1743.

Fisher, H. (2000). El primer sexo. Madrid: Santillana.

Fundación Nacional para el Desarrollo Integral del Menor (2005). Instalación del nuevo currículum en Jardines de la Fundación. Santiago: Integra.

Garaigordobil Landazabal, M. (2005). Conducta antisocial durante la adolescencia: Correlatos socioemocionales, predictores y diferencias de género. Psicología Conductual, vo. 13, n. 2, 197-215. 
Gintis, H. (2007). A framework for the unification of the behavioral sciences. Behavioral and Brain Sciences, vol. 30, n. 1, 1-16.

Grimm, H. (2003). Störungen der Sprachentwicklung. Hogrefe: Göttingen.

Herrera, M.O., Mathiesen, M.E. y Pandolfi, A.M. (2000). Variación en la competencia léxica del preescolar: algunos factores asociados. Estudios Filológicos, n. 35, 61-70.

Herrera, M.O., Mathiesen, M.E., Morales, M., Proust, P. y Vergara, M. (2006). Actitud del adulto a cargo de la sala cuna hacia el género y su relación con la calidad del ambiente educativo. Estudios Pedagógicos, vol. 32, n. 1, 7-19.

Hyde, J.S. y Mertz, J.E. (2009). Gender, culture, and mathematics performance. PNAS, vol. 106, n. 22, 8801. Web: http://www.pnas.org/content/106/22/8801, (07/2011).

Inderbitzen, H. y Harbin, C. (1992). An investigation of the Construct Validity of de Teenage Inventatory of Social Skills: A Corvengent Approach. Trabajo presentado en la Junta Anual de la Asociación por el Avance de la Terapia de Comportamiento, Boston, USA.

Inglés, C., Benavides, G., Redondo, J., García-Fernández, J., Ruiz-Esteban, C., Estévez, C. y Huescar, E. (2009). Conducta prosocial y rendimiento académico en estudiantes españoles de Educación Secundaria Obligatoria. Anales de psicología, vol. 25, n. 1., 93-101.

Kanazawa, S. y Vandermassen, G. (2005). Engineers have more sons, nurses have more daughters. An evolutionary psychological extension of Baron-Cohen's extreme male brain theory of autism and its empirical implications. Journal of Theoretical Biology, vol. 233, n. 4, 589-99.

Lera, M. J., y Sánchez, V. (2001). Y colorín colorado, este cuento se ha acabado. Género e interacciones contando un cuento. Infancia, $n$. 69, 27-31.

Mathiesen, M. E., Merino, J.M., Castro, G., Mora, O. y Navarro, G. (2011). Adaptación socioemocional en escolares: evaluación de un instrumento nuevo en la provincia de Concepción. Estudios Pedagógicos, vol. 37, n. 2, 61-75.

Mathiesen, M. E., Merino J.M., Mora, O., Navarro, G. y Castro, G. (2010-2011). Incubando capital humano regional: evaluación longitudinal de un programa extracurricular universitario para estudiantes de establecimientos municipales de alto potencial académico. Proyecto Fondecyt 1100260.

Miller, A. y Kazanawa, S. (2007). Why beautiful people have more daughters? New York: Penguin.

Morales, M., Mathiesen De G., M.E. y Navarro, G. (2008). Diferencia entre el desarrollo de niños y el desarrollo de niñas que asisten a primer ciclo de educación parvularia en la provincia de Concepción. ¿Las niñas se desarrollan igual que los niños? Investigaciones en Educación, vol. $8, n .1,85-100$.

Nyborg, H. (2005). Sex-related differences in general intelligence g, brain size, and social status. Personality and Individual Differences, vol. 39, n. 3, 497-509.

Organización de las Naciones Unidas para la Educación, la Ciencia y la Cultura. (2004). Educación para todos: ¿Va el mundo por buen camino? Madrid: UNESCO.

Organización de las Naciones Unidas para la Educación, la Ciencia y la Cultura. (2003). Gender and education for all: The leap to equality. Paris: UNESCO.

Programa de Promoción de la Reforma Educativa en América Latina y el Caribe (2004). Etnicidad, Raza, Género y Educación en América Latina. Santiago: PREAL.

Raven, J. C. (2003). Test de Matrices Progresivas. Buenos Aires: Editorial Paidós.

Roa, R. (2010). Influencia de la conducta prosocial en el desarrollo educativo y social de alumnos de octavo básico de la comuna de Concepción. Memoria. Concepción: Universidad de Concepción, Escuela de Sociología.

Robinson, J. y P. Shaver (1991). Measures of personality and social psychological attitudes. San Diego, California: Academic Press. Hardcout Brace Jovanovich. Publishers.

Steinmayr, R. y Spinath, B. (2009). What Explains Boys' Confidence in their Intelligence? Sex Roles, vol. 61, n. 9-10, 736-749. 
